\title{
Inertial frame rotation induced by rotating gravitational waves
}

\author{
Donald Lynden-Bell ${ }^{1 *}$ Jiří Bičák ${ }^{2,1 \dagger}$ and Joseph Katz ${ }^{3,1 \ddagger}$ \\ ${ }^{1}$ Institute of Astronomy, Madingley Road, Cambridge CB3 OHA, United Kingdom \\ ${ }^{2}$ Institute of Theoretical Physics, Charles University, 18000 Prague 8, Czech Republic \\ ${ }^{3}$ The Racah Institute of Physics, Givat Ram, 91904 Jerusalem, Israel
}

November 5, 2018

\begin{abstract}
We calculate the rotation of the inertial frame within an almost flat cylindrical region surrounded by a pulse of non-axially-symmetric gravitational waves that rotate about the axis of our cylindrical polar coordinates. Our spacetime has only one Killing vector. It is along the $z$-axis and hypersurface orthogonal. We solve the Einstein equations to first order in the wave amplitude and superpose such linearized solutions to form a wave pulse. We then solve the relevant Einstein equation to second order in the amplitude to find the rotation of inertial frames produced by the pulse. The rotation is without time delay.

The influence of gravitational wave angular momentum on the inertial frame demonstrates that Mach's principle can not be expressed in terms of the influence of the stressenergy-momentum tensor alone but must involve also influences of gravitational wave energy and angular momentum.
\end{abstract}

PACS numbers 04.20.Jb 04.30. - -

*email: dlb@ast.cam.ac.uk

${ }^{\dagger}$ email:bicak@mbox.troja.mff.cuni.cz

¥email:jkatz@phys.huji.ac.il 


\section{Introduction}

Mach [21] rejected Newton's [26] absolute space and suggested that some average of the positions and motions of the masses in the Universe determines the inertial frame.

Einstein [10], 11] was inspired by Mach's principle and his earlier work [9] showed that the local inertial frame was not absolute but was affected by the rotation and acceleration of matter nearby. Extrapolating from this he said, in essence, the relativity of inertia is not fulfilled if inertia is affected by the presence of matter it must be entirely caused by it. He also said there is no inertia of mass against space but only inertia of mass against mass. Thus he hoped that the inertial frame might be determined by the distribution of the stress tensor ${ }^{1}$ $T_{\mu \nu}$. His efforts to remove the external influence of the boundary conditions at infinity on the solutions of his equations led him to consider closed spaces with no spatial boundaries [10]. Here we show that within General Relativity, any general statement of Mach's principle that attributes all dragging of inertial frames solely to the distribution of $T_{\mu \nu}$ as the origin of inertia is false (see [5]). One counter example suffices to demonstrate this. We find that there is an almost flat cylindrical region near the axis of a revolving gravitational wave pulse (which inevitably has no $T_{\mu \nu}$ ) and demonstrate that the inertial frame in the cylindrical interior rotates relative to the inertial frame at great distances.

The exact cylindrical wave solutions [2], [12, [7], 30] carry no angular momentum about the axis, because their wave fronts have normals with no component around the axis. Bondi [6] emphasises this point.

In a sense our work pushes at an open door, since now all accept that gravitational waves exist and carry both energy and angular momentum. This is not merely known theoretically, but also observationally [28], 27], cf. also [24] and [20].

It would be astonishing if that energy and angular momentum did not produce gravity and rotation of the inertial frame. Indeed, as we described in more detail in paper [4], the work of Corvino [8] demonstrates that this must be the case and in [4] we have explicitly calculated the gravity of a non-linear pulse of gravitational waves.

Here our aim is to produce a nice clean example of the rotation of the inertial frame in an almost flat region surrounded by rotating gravitational waves.

\footnotetext{
${ }^{1}$ Indices $\lambda, \mu, \nu, \rho, \cdots=0,1,2,3$; indices $k, l, m, n \cdots=1,2,3$ and $a, b, c, \cdots=0,1,2$. The metric $g_{\mu \nu}$ has signature +--- and $g$ is its determinant. Covariant derivatives are indicated by a $D$, partial derivatives by a $\partial$ and covariant derivatives in a three subspace by $\nabla_{a}$ or $\nabla_{k}$. The permutation symbol in 4 dimensions is $\epsilon_{\mu \nu \rho \sigma}$ with $\epsilon_{0123}=1$ and in 3 dimensions $\epsilon_{k l m}$ with $\epsilon_{123}=1$. Finally $\eta_{\mu \nu \rho \sigma}=\sqrt{-g} \epsilon_{\mu \nu \rho \sigma}$.
} 
An extreme example of inertia due to gravitational waves alone is provided by Gowdy's [15] universe, a closed world that contains nothing except gravitational waves. One of our ultimate aims is to discuss the meaning of Mach's principle in General Relativity and particularly its application to such systems. However, such applications and the question as to what bearing Mach's principle has on the existence of dark energy are outside the scope of this paper.

When we first discussed Mach's principle in FRW universes via perturbation theory [18], we pointed out that our first order perturbation theory inevitably missed the second order contribution expected from gravitational waves (see also our recent comprehensive paper [3]). Here we show that there must be such a contribution.

We consider waves in spaces that have a hypersurface orthogonal Killing vector along $O z$. If such waves have axial symmetry their wave normals converge on the axis and they carry no angular momentum. To get truly rotating waves that carry angular momentum around the axis, we can not impose axial symmetry. As no exact non-axisymmetric rotating wave solutions are known, we work via perturbation theory. First we solve the flat space wave equation for linearized gravitational waves with symmetry along $O z$, using cylindrical polar coordinates. Then we superpose these linear solutions to form a wave pulse that rotates around the axis. This pulse generalizes to non-axial-symmetry the wave pulse found by Bonnor [7] and by Weber and Wheeler [30]. In the spacetime generated by such a pulse of linear waves, we then solve one of Einstein's equations to second order in the wave amplitude. Following [1] and 4], we see that the wave pulse generates an "effective source term" of second order in the wave amplitude. This means that the first order changes in the metric due to the wave can be neglected when calculating the extra perturbation due to these effective sources. (Keeping the changes could only lead to terms of third order in the wave amplitude.) Thus we have to calculate in flat space the effects of sources that are quadratic in the wave amplitude. Such perturbative techniques are well discussed in [14].

\section{Calculations}

We consider as in [4] source free metrics with one hypersurface orthogonal Killing vector of spacelike translations $\boldsymbol{\zeta}$. Coordinates can be chosen so that $z=x^{3}$ and

$$
\left\{x^{\mu}\right\}=\left\{x^{a}, x^{3}\right\} \quad \text { with } \quad a=0,1,2 \quad \text { that } \quad \zeta=\{0,0,0,1\}
$$


and the metric can be written in the form introduced by Ashtekar, Bičák and Schmidt [1]:

$$
d s^{2}=e^{-2 \psi} g_{a b} d x^{a} d x^{b}-e^{2 \psi} d z^{2}
$$

$\psi$ and $g_{a b}$ are functions of the $x^{c}$ only. The source-free Einstein's equations $R_{\mu \nu}=0$ take then the following form in terms of the Ricci tensor $\mathcal{R}_{a b}$ of the $2+1$-space $d \sigma^{2}=g_{a b} d x^{a} d x^{b}$ :

$$
\begin{aligned}
& R_{a b}=0 \quad \Rightarrow \quad \mathcal{R}_{a b}=2 \partial_{a} \psi \partial_{b} \psi \\
& R_{33}=0 \quad \Rightarrow \quad g^{a b} \nabla_{a} \nabla_{b} \psi=0 .
\end{aligned}
$$

Because $\psi$ satisfies (2.4), the right-hand side of (2.3) automatically satisfies the Bianchi identities in $2+1$ spacetime. To zero order $g_{a b}$ reduces to the flat spacetime metric and we choose cylindrical coordinates $x^{0}=t, x^{1}=\rho, x^{2}=\varphi$, so, in the notations of [4] where a dot means a derivative with respect to $t$ and a prime a derivative with respect to $\rho$, our linearized waves obey the wave equation

$$
\ddot{\psi}-\psi^{\prime \prime}-\frac{1}{\rho} \psi^{\prime}-\frac{1}{\rho^{2}} \partial_{\varphi}^{2} \psi=0
$$

The solutions are expressed in Bessel functions $J_{m}(\omega \rho)$ :

$$
\psi=A e^{ \pm i m \varphi \pm i \omega t} J_{m}(\omega \rho)
$$

We note that the special class of these waves with $m=0$ constitute the exact solutions mentioned in the Introduction. We follow [7] and [30] in choosing a superposition of such waves but in order to have rotating linearized waves we need $m \neq 0$ and we choose waves of the form $e^{i(m \varphi-\omega t)}$ with $m \omega>0$ so that the wave fronts rotate positively about the $z$ axis. Our linear wave pulse is given by the superposition with $a>0$ being the effective duration of the pulse,

$$
\psi=B \int_{0}^{\infty}(a \omega)^{m} e^{-a \omega} e^{i(m \varphi-\omega t)} J_{m}(\omega \rho) a d \omega+c . c .
$$

$B$ is a dimensionsless amplitude. It is convenient to define $\alpha=\alpha(t)=a+i t$. Thanks to the Bateman Manuscript Project of Erdélyi et al [13, formula 8.6.5, we find

$$
\psi=B a^{m+1} e^{i m \varphi} \cdot 2^{m} \frac{\Gamma\left(m+\frac{1}{2}\right)}{\sqrt{\pi}} \cdot \rho^{m}\left(\alpha^{2}+\rho^{2}\right)^{-m-\frac{1}{2}}+c . c .
$$

$\psi$ may be written in terms of non-dimensional variables as in [4],

$$
\tilde{\rho}=\frac{\rho}{a} \quad, \quad \tilde{t}=\frac{t}{a}, \quad \text { and with } \quad 2^{m} \frac{\Gamma\left(m+\frac{1}{2}\right)}{\sqrt{\pi}}=(2 m-1) ! !
$$


$\psi$ can also be written in real terms as follows:

$$
\psi=2 B(2 m-1) ! ! \frac{\tilde{\rho}^{m} \cos \left[m \varphi-\left(m+\frac{1}{2}\right) \chi\right]}{\left[\left(1+\tilde{\rho}^{2}-\tilde{t}^{2}\right)^{2}+4 \tilde{t}^{2}\right]^{\frac{1}{2}\left(m+\frac{1}{2}\right)}}, \quad \text { where } \quad \chi=\arctan \frac{2 \tilde{t}}{1+\tilde{\rho}^{2}-\tilde{t}^{2}} .
$$

So the phase of $\psi$ is $m \varphi-\left(m+\frac{1}{2}\right) \chi$. The $\tilde{\rho}$-dependence of this phase gives spirality to the wave and changes sign at $t=0$. Figure 1 shows the profile of $\psi$ at different times for $m=10$ and $\varphi=0$.

To calculate the source term on the right-hand side of 2.3 we need derivatives of expression 2.8. . In particular the $t, \varphi$ component of the source will be given by $2 \dot{\psi} \partial_{\varphi} \psi$. This product contains two terms that are independent of $\varphi$ and two terms that vary like $\sin 2 m \varphi$ or $\cos 2 m \varphi$. Those terms can not cause any rotation of the inertial frame on the axis. We therefore concentrate on the terms that are independent of $\varphi$ and denote them by the averaging symbol $<>$, implying an average over $\varphi$. Defining $j$, we find from (2.8) and 2.9),

$$
j=-2<\dot{\psi} \partial_{\varphi} \psi>=\frac{2 B^{2}}{a} m(2 m+1)[(2 m-1) ! !]^{2} \tilde{\rho}^{2 m} Q^{-m-3 / 2}\left(\frac{\partial Q}{\partial u}+4 \tilde{t}^{2}\right),
$$

in which

$$
u=\tilde{\rho}^{2} \quad \text { and } \quad Q=\left(1+u-\tilde{t}^{2}\right)^{2}+4 \tilde{t}^{2} .
$$

Before proceeding further we return to equations (2.3), now regarding the right-hand side as a known second order source of perturbation of our now first order metric. As the source terms are already of second order, we may neglect the change of the metric from flat space in calculating their effects, as such changes could only give effects of third order. We can write the fully perturbed metric in the form (2.2) in which, see (5.4) in [4],

$$
g_{a b} d x^{a} d x^{b}=e^{2 \gamma}\left(d t^{2}-d \rho^{2}\right)-W^{2}(d \varphi-\omega d t)^{2} .
$$

The flat space equation for the axially symmetrical part of $\omega$ is then, cf. (5.10) in [4,

$$
<\mathcal{R}_{02}>=\frac{1}{2 \rho}\left(\rho^{3}<\omega>^{\prime}\right)^{\prime}=2<\dot{\psi} \partial_{\varphi} \psi>=-j .
$$

We first integrate once with the boundary condition that $\langle\omega\rangle$ is not singular on the axis:

$$
\rho^{3}<\omega>^{\prime}=-\int_{0}^{\tilde{\rho}^{2}} j d \rho_{1}^{2} .
$$

Using the boundary condition that $\langle\omega\rangle \rightarrow 0$ at infinity we then find that

$$
<\omega>=\frac{1}{2} \int_{\tilde{\rho}^{2}}^{\infty} \frac{1}{\rho_{2}^{4}}\left(\int_{0}^{\tilde{\rho}^{2}} j d \rho_{1}^{2}\right) d \rho_{2}^{2}
$$


where $\rho_{1}^{2}$ and $\rho_{2}^{2}$ are the dummy variables. Doing the outer integration by parts, we deduce

$$
<\omega>=\frac{1}{2 u} \int_{0}^{u} j d u+\int_{u}^{\infty} \frac{1}{2 u} j d u .
$$

Substituting 2.11) into 2.17, we get, after integrating the $Q^{-m-3 / 2}(\partial Q / \partial u)$ terms by parts,

$$
<\omega>=\frac{2 B^{2}}{a} m[(2 m-1) ! !]^{2}\left[\frac{m}{u} I_{m-1}+2(2 m+1) \frac{\tilde{t}^{2}}{u} I_{m}+(m-1) H_{m-1}+2(2 m+1) \tilde{t}^{2} H_{m}\right],
$$

in which

$$
I_{m}(u)=\int_{0}^{u} u^{m} Q^{-m-3 / 2} d u \quad, \quad H_{m}(u)=\int_{u}^{\infty} u^{m-1} Q^{-m-3 / 2} d u .
$$

The evaluation of $\langle\omega\rangle$ is thus reduced to the calculation of $I_{m}$ and $H_{m}$ for all $m$. This is done in the Appendix.

\section{Rotation of inertial frames at small and great distances}

On axis the first two terms in the large brackets of 2.18 are equal to zero. So using A.15 with $b=1-\tilde{t}^{2}$ and $c=1+\tilde{t}^{2}$ we get from 2.18

$$
<\omega>_{0}=\frac{B^{2}}{a} \cdot \frac{(2 m) !}{2^{2 m-1}} \cdot \frac{1+m\left(1+\tilde{t}^{2}\right)}{\left(1+\tilde{t}^{2}\right)^{2}} .
$$

This is greatest at $\tilde{t}=0$ exactly. Notice that there is no time lag between the wave arriving
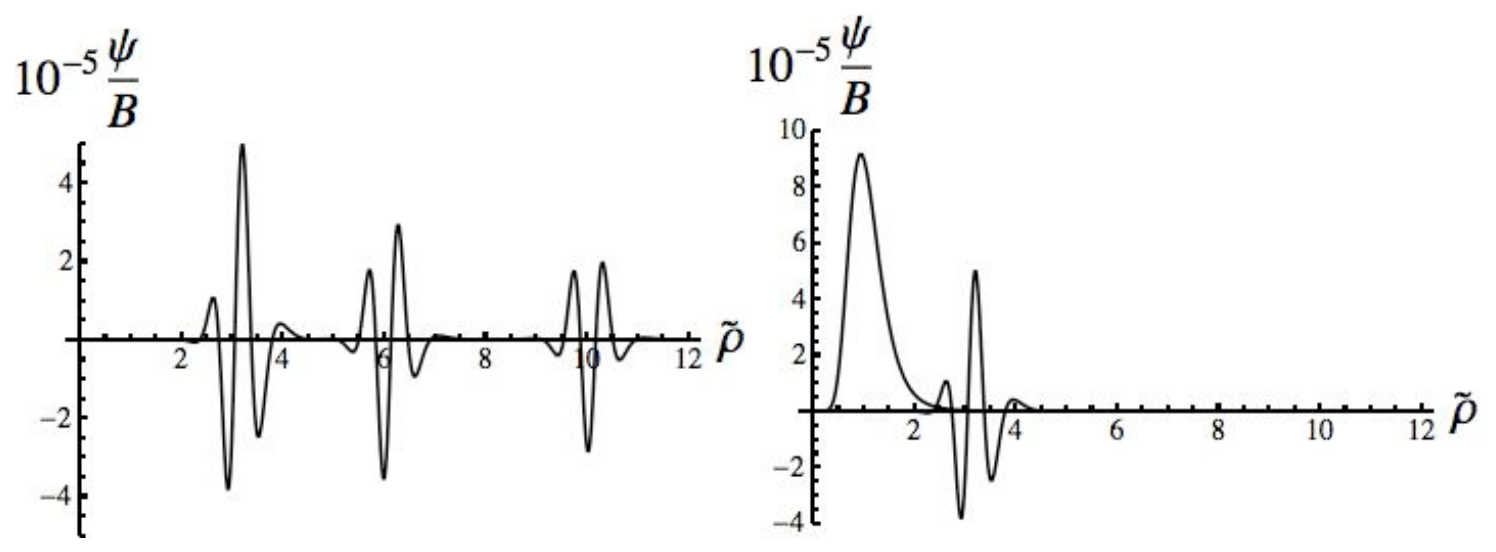

Figure 1: This shows $\psi / B$, the wave profiles for $m=10$ and $\varphi=0$ at 5 different times at adapted scales. On the left-hand side $\psi / B$ is shown as it circulates inwards, from right to left at times $\tilde{t}=-10,-6$, and -3 . The right-hand side shows it as it circulates at closest approach to the axis $\tilde{t}=0$. Notice there is very little closer than $\tilde{\rho} \simeq 0.4$ even at $\tilde{t}=0$. Superposed on the right-hand side is the profile as the wave starts to retreat at $\tilde{t}=3$. Thereafter the profiles are the same as on the left-hand side of the figure but in reverse order. At all times the wave profile centers around $\tilde{\rho}=\sqrt{1+\tilde{t}^{2}}$.

closest to the axis - most of the energy never gets nearer than $\tilde{\rho} \simeq 0.4$ (see Figure 1 at 
$\tilde{t}=0)$ - and the resulting rotation of inertial frames. Just as in the work of Lindblom and Brill [16] on inertia induction from falling rotating spheres, the non-local effect appears instantaneously when suitable Machian coordinates (gauge) are chosen. This is a consequence of Mach's principle being embodied in the "instantaneous" constraint equations of General Relativity. In electricity, the presence of a charge within a sphere at time $t$ can be ascertained "instantaneously" by measuring the flux of its field out of a surrounding surface. You do not have to wait for the field to come out from the charge - it is there already.

Returning to our inertial frame on the axis, a rod lying peacefully at the origin, initially oriented at $\varphi=\varphi_{0}$, will remain in the flat space near there and feel no torque, but, due to the rotation of the inertial frame it will point towards $\varphi=\phi(t)$ where

$$
\phi(t)=\phi(0)+\int_{-\infty}^{t}\left\langle\omega>_{0} d t=\phi(0)+B^{2} \frac{(2 m+1) !}{2^{2 m}}\left[\arctan \tilde{t}+\frac{\pi}{2}+\frac{\tilde{t}}{(2 m+1)\left(1+\tilde{t}^{2}\right)}\right] .\right.
$$

When the whole pulse has passed it ends up oriented towards

$$
\phi(\infty)=\phi(0)+B^{2} \frac{(2 m+1) !}{2^{2 m}} \cdot \pi .
$$

For symmetry we choose

$$
\phi(0)=-B^{2} \frac{(2 m+1) !}{2^{2 m}} \cdot \frac{\pi}{2}
$$

so that $\phi=0$ when $\tilde{t}=0$ and

$$
\phi(t)=B^{2} \frac{(2 m+1) !}{2^{2 m}}\left[\arctan \tilde{t}+\frac{\tilde{t}}{(2 m+1)\left(1+\tilde{t}^{2}\right)}\right] .
$$

Figure 2 shows $\phi(t)$ for $t \geq 0$. It is antisymmeric about $t=0$.

Far from the axis we have from 2.18 and $(\mathrm{A} .9$

$$
<\omega>\simeq \frac{B^{2}}{a} \cdot \frac{m(m !)(2 m-1) ! !}{2^{m-1}} \cdot \frac{1}{\tilde{\rho}^{2}} \quad, \quad \tilde{\rho} \gg 1 .
$$

As expected this is constant in time for $\tilde{\rho}$ large, but falls off like $\tilde{\rho}^{-2}$ as befits a cylindrical system with a total angular momentum per unit height, cf. [4],

$$
J=\frac{\pi}{\kappa} a B^{2} \cdot \frac{m(m !)(2 m-1) ! !}{2^{m-2}} .
$$

For general $\tilde{t}$ and $\tilde{\rho},\langle\omega\rangle$ is given in $(2.18)$ with $I_{m}$ and $H_{m}$ from $($ A.6 6 and $($ A.12). But for $\tilde{\rho}^{2} \ll 1+\tilde{t}^{2}$ we need to show that our space is almost flat so that $\langle\omega\rangle$ can be properly interpreted as a rotation of the inertial frame. We therefore evaluate $\langle\omega>$ not just at $\tilde{\rho}=0$, 


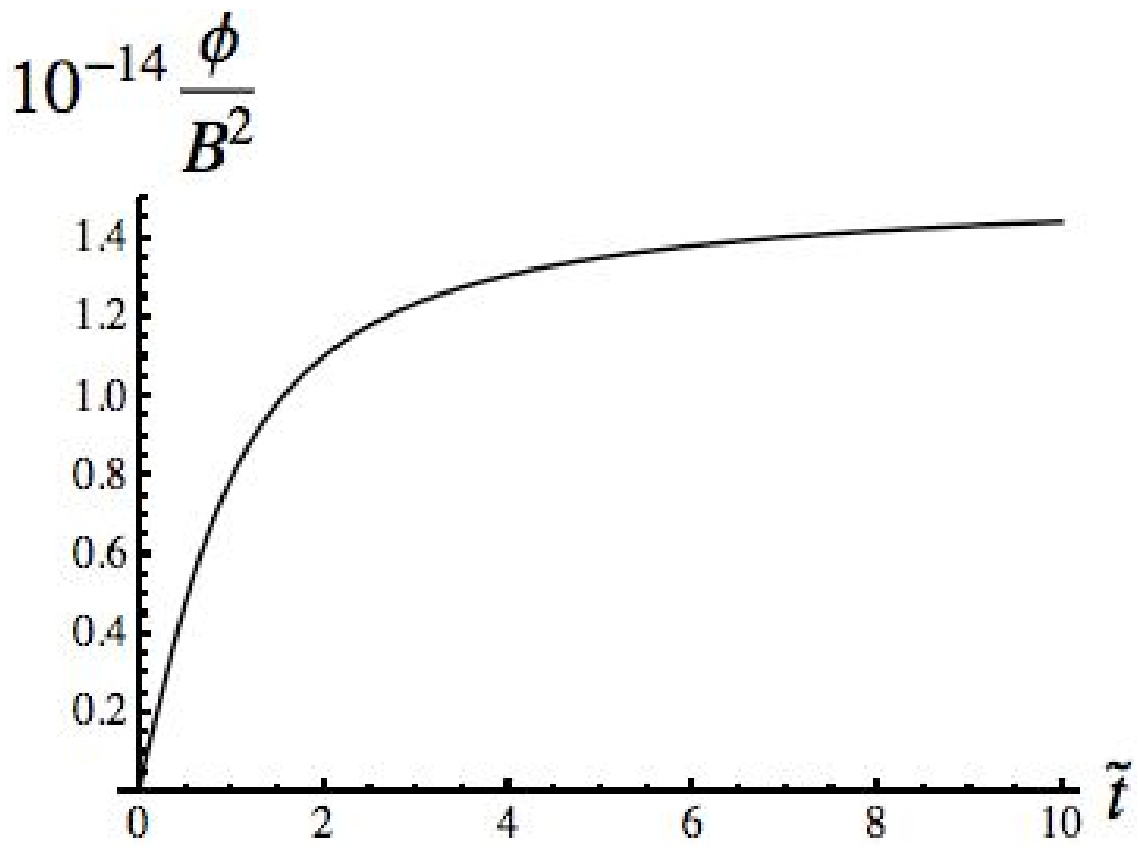

Figure 2: The angle $\phi(\tilde{t}) / B^{2}$ through which the inertial frame at the origin turns as a function of time for $m=10$. The initial angle before the wave comes in, is chosen as $-\phi_{0}$ so that $\phi$ is zero at $\tilde{t}=0$. This produces anti-symmetry about $\tilde{t}=0 . \omega(\tilde{t}, 0)$ is given by the gradient of the curve. It is greatest at $\tilde{t}=0$.

but in this larger region, and show that at each time $\langle\omega\rangle$ varies weakly with $\tilde{\rho}$ there. Indeed from A.17 and A.18

$$
<\omega>\simeq<\omega>_{0}\left[1-\frac{(2 m-1) ! !}{(m+1) !} \cdot \frac{1}{\left(1+\tilde{t}^{2}\right)^{m+1}} \cdot \frac{(2 m-5)+(2 m+7) \tilde{t}^{2}}{(m+1)+m \tilde{t}^{2}} \cdot\left(\frac{\tilde{\rho}^{2}}{1+\tilde{t}^{2}}\right)^{m}\right] .
$$

Figures 3 and 4 show $j, \kappa$ times the angular momentum density, and the resulting $<\omega(t, \rho)>$.

The fact that the source terms in $(2.3)$ all vary as $\tilde{\rho}^{2 m}$ shows that the other terms in the metric will vary similarly, so wherever $\left[\tilde{\rho}^{2} /\left(1+\tilde{t}^{2}\right)\right]^{m}$ is negligible our second order metric will take the form

$$
d s^{2}=e^{2 \psi}\left[d t^{2}-d \rho^{2}-\rho^{2}\left(d \varphi-<\omega>_{0} d t\right)^{2}\right]-e^{-2 \psi} d z^{2} \quad, \quad \tilde{\rho}^{2} \ll\left(1+\tilde{t}^{2}\right)
$$

with $\psi$ given by $(2.10)$. If we now go to rotating axes by writing $\tilde{\varphi}=\varphi-\phi(t)$, then our metric becomes

$$
d s^{2}=e^{2 \psi}\left(d t^{2}-d \rho^{2}-\rho^{2} d \tilde{\varphi}^{2}\right)-e^{-2 \psi} d z^{2} \quad, \quad \tilde{\rho}^{2} \ll\left(1+\tilde{t}^{2}\right)
$$

which is flat in the reduced space. Furthermore the wave itself never penetrates significantly within $\tilde{\rho} / \sqrt{1+\tilde{t}^{2}} \simeq 0.4$. 


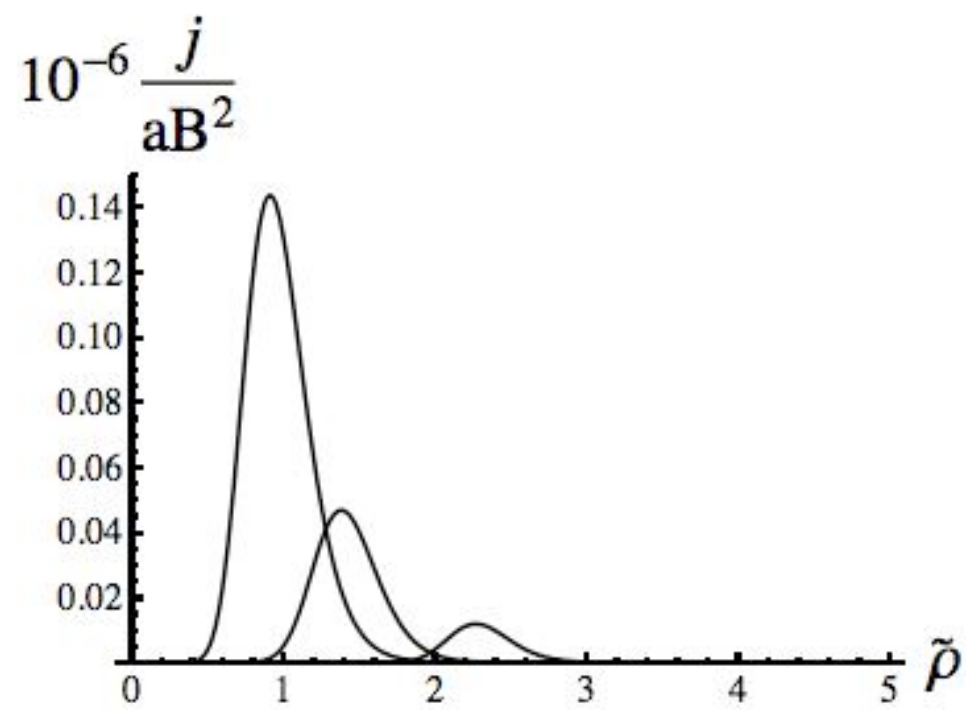

Figure 3: The profile of the angular momentum density at $t \simeq 0,1$ and 2. Again the drawing is for $m=10$.

\section{Angular momentum transport by gravitational torques}

Those who have studied angular momentum transport in spiral galaxies, know that the classical gravitational stress tensor is [17, [25]

$$
\sigma_{k l}=\frac{1}{\kappa}\left(2 \partial_{k} \psi \partial_{l} \psi-\delta_{k l} \sum_{m}\left|\partial_{m} \psi\right|^{2}\right)
$$

where here $\psi$ is the classical gravitational potential. The gravitational couple transfering angular momentum outwards through a cylinder of radius $\rho$ is

$$
C_{g}=\int \epsilon_{3 k l} X^{k}\left(\sigma^{l m} d S_{m}\right)
$$

where $d \vec{S}$ is the outward pointing surface element and $\vec{X}$ the radius vector from the axis. Evidently this reduces to

$$
C_{g}=\frac{2}{\kappa} \int \partial_{\rho} \psi \partial_{\varphi} \psi \rho d \varphi d z
$$

For these gravitational stresses to carry angular momentum outwards there must be a positive correlation between $\partial_{\rho} \psi$ and $\partial_{\varphi} \psi$ averaged over such a cylinder. Such a correlation gives a trailing sense of spirality to contours of constant $\psi$ in the sense that the outer parts of a spiral galaxy trail that of the inner parts in the sense defined by the rotation. It is no different here; for angular momentum transport outward, the spiral formed by contours of $\psi$ must trail, but contrarywise when they form a leading spiral with the outside further advanced than the inside, the angular momentum is transported inward. Thus our knowledge of spiral 


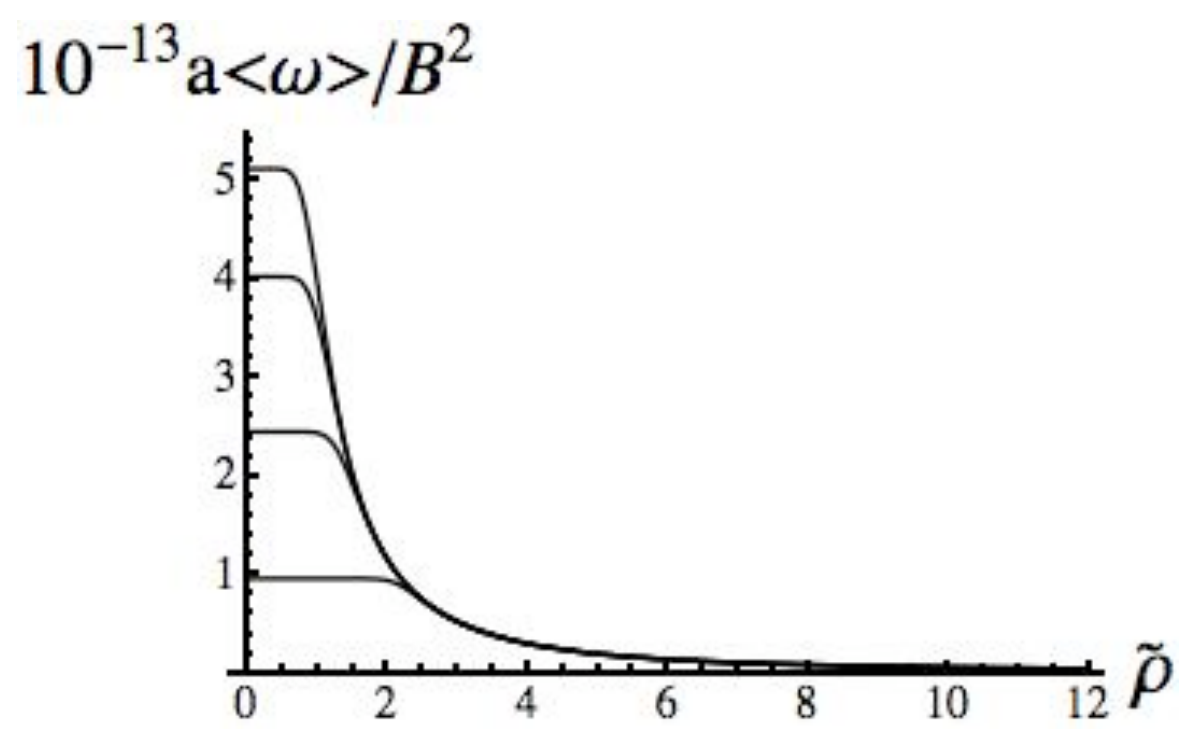

Figure 4: The profile $\langle\omega\rangle$ as a function of $\tilde{\rho}$ at selected times $\tilde{t}=0,0.5,1$, and 2 . Notice the still flat uniformly rotating central region when $\tilde{t}=0$.

structure in galaxies enabled us to predict that there would be leading spirals when the wave was going in and therefore transporting its angular momentum inward, but trailing spirals when our gravitational wave was receding from the axis. At $t=0$ there should be no angular momentum transport so the contours of $\psi$ should form a rotating cartwheel structure with no spirality. These expectations were beautifully confirmed when we plotted the contours of $\psi$ using Mathematica. The waves always rotate counterclockwise in Figure 5. They start as quite tightly wrapped leading spirals; as they move inwards they keep rotating but the wrapping becomes less tight. At $t=0$ they unwrap to form a rotating cartwheel structure and thereafter they move outward as trailing spirals carrying angular momentum with them. It is when they are closest in that their rotation causes the greatest rotation of the inertial frame in the central region, though the waves themselves never reach much further in than $\tilde{\rho}=1$. Nevertheless the second order effect of the angular momentum causes the rotation of the inertial frame within. This is illustrated by the direction of the central arrow that shows the orientation of a rod initially at rest at the origin. Because this effect is second order, the rotation seen in the figure is dependent on the amplitude of the wave, whereas the spiral shapes are not. 


\section{$5 \quad$ Energy and angular momentum}

In Section 3 we used the asymptotic form of $\langle\omega\rangle$ to deduce the total angular momentum per unit height but we could also calculate it via the angular momentum density $j / \kappa$

$J=\frac{1}{\kappa} \int_{0}^{\infty} j 2 \pi \rho d \rho=\frac{4 \pi}{\kappa} a B^{2} m[(2 m-1) ! !]^{2}\left[m I_{m-1}+(2 m+1) \tilde{t}^{2} I_{m}\right]=\frac{\pi}{\kappa} a B^{2} \frac{m(m !)(2 m-1) ! !}{2^{m-3}}$.

Any quantity $\psi$ that obeys $\square \psi=0$ automatically conserves both $J$ and the quantity

$$
\frac{1}{\kappa} \int\left(\dot{\psi}^{2}+|\vec{\nabla} \psi|^{2}\right) d V
$$

The identity

$$
\frac{1}{\kappa} \partial_{t}\left(\dot{\psi}^{2}+|\vec{\nabla} \psi|^{2}\right)=\frac{1}{\kappa} \vec{\nabla} \cdot(2 \dot{\psi} \vec{\nabla} \psi)
$$

follows directly from the wave equation and can be interpreted as a conservation law with $\frac{2}{\kappa} \dot{\psi} \vec{\nabla} \psi \cdot d \vec{S}$ being the flux through an element of the closed surface surounding a volume $V$. We notice that the flux vector now directed through a surface $\varphi=$ const will on multiplication by $\rho$ give the angular momentum density $j / \kappa$. Integrating $\frac{1}{\kappa}\left(\dot{\psi}^{2}+|\vec{\nabla} \psi|^{2}\right)$ over a volume of unit height we find the grand total and using the formulæ of the Appendix,

$$
\int_{0}^{\infty} \int_{0}^{2 \pi} \frac{1}{\kappa}\left(\dot{\psi}^{2}+|\vec{\nabla} \psi|^{2}\right) d \varphi \rho d \rho=\frac{\pi}{\kappa} B^{2} \frac{m !(2 m+1) ! !}{2^{m-1}}
$$

which is independent of time as expected. We may identify this conserved quantity with energy per unit length via the following argument. The conservation laws $\nabla_{b} \mathcal{T}_{a}^{b}=0$, see [4], in the reduced $2+1$ space follow from its contracted Bianchi identities of which the $x^{0}=t$ one reads

$$
\frac{1}{\rho}\left[\left(\rho \mathcal{T}_{0}^{0}\right)^{\cdot}+\left(\rho \mathcal{T}_{0}^{1}\right)^{\prime}+\partial_{\varphi}\left(\rho \mathcal{T}_{0}^{2}\right)\right]=0 .
$$

Ordinary derivatives in flat space have replaced covariant ones because $\mathcal{T}_{a}^{b}$ is already of second order in the wave amplitude:

$$
\kappa \mathcal{T}_{a b}=\mathcal{R}_{a b}-\frac{1}{2} g_{a b} \mathcal{R}=2 \partial_{a} \psi \partial_{b} \psi-g_{a b}\left(\dot{\psi}^{2}-|\vec{\nabla} \psi|^{2}\right)
$$

where $\vec{\nabla} \psi$ is the normal flat space gradiant in $\rho$ and $\varphi$,

$$
|\vec{\nabla} \psi|^{2}=\psi^{\prime 2}+\frac{1}{\rho^{2}}\left(\partial_{\varphi} \psi\right)^{2} .
$$

From (5.6) we see that

$$
\kappa \mathcal{T}_{0}^{0}=\dot{\psi}^{2}+|\vec{\nabla} \psi|^{2}
$$


which is the density of our conserved quantity. The total energy per unit length as measured from the conicity at infinity, $\gamma_{\infty}$, was shown to be $2 \pi \gamma_{\infty} / \kappa$ by [1].

In [4], eq. (5.31), we show that $2 \pi \gamma_{\infty} / \kappa$ is given by the left-hand side of (5.4). This completes the identification of our conserved quantity with the energy per unit height. It also shows that the wave produces a long range effect on $\gamma$ far from the region $\rho \simeq t$ where its main amplitude lies. It is one of the strange properties of cylindrical General Relativity that this conicity is independent of $\rho$ outside the wave. This has the effect that while particle trajectories are globally bent by the defect angle nevertheless there are no orbits along finite sections of circles (about the axis) caused by the long distance gravity of the cylindrical wave. Rather the geodesics are like the geodesics on a cone all of which go to infinity. It would be interesting to calculate the gravity due to a more realistic three dimensional wave pulse for which the odd behaviour at infinity found in $2+1$ dimensions would not obscure the interpretation [19].

\section{Acknowledgements}

J.B. and J.K. are grateful to the Institute of Astronomy, Cambridge University, and the Royal Society for hospitality and support. J.B. also acknowledges the hospitality of the Albert Einstein Institute in Golm and the Institute of Theoretical Physics at FSU in Jena, the support of the Alexander von Humbolt Foundation, the partial support from the Grant GAČR 202/06/0041 of the Czech Republic, of Grant No LC06014 and MSM0021620860 of the Ministry of Education and from SFB/TR7 in Jena.

\section{References}

[1] Ashtekar A, Bičák J and Schmidt B G 1997 Asymptotic structure of symmetry reduced General Relativity Phys. Rev. D 55669 (Reprint gr-qc/9608042)

[2] Beck G 1925 Zur Theorie binärer Gravitationsfelder Zeit. Phys. 33713

[3] Bičák J, Katz J and Lynden-Bell D 2007 Cosmological perturbation theory, instantaneous gauges, and local inertial frames Phys. Rev D 76063501 (Reprint arXiv:grqc/0803.4106)

[4] Bičák J, Katz J and Lynden-Bell D 2007 Gravitational waves and dragging effects Class. Quantum Grav. ? ? (Reprint gr-qc/?) 
[5] Bondi H 1952 Cosmology (Cambridge:CUP)

[6] Bondi H 1994 The Angular momentum of cylindrical systems in General Relativity Proc. R. Soc. Lond. A 44657

[7] Bonnor W B 1957 Non-singular fields in General Relativity J. Math. E6 Mech. 6203

[8] Corvino J 2000 Scalar Curvature Deformation and a Gluing Construction for the Einstein Constraint Equations Commun. Math.Phys. 214137

[9] Einstein A 1913 Letter to Mach in Collected papers Vol. $5 \mathrm{n}^{0}$ 448, p. 340 (Princeton: PUP) 1995

[10] Einstein A 1917 Cosmological considerations on the general theory of relativity - translated by Perrett W and Jeffery G B in The principle of relativity (New York: Dover Publ.)

[11] Einstein A 1950 The meaning of relativity $3^{\text {rd }}$ Ed. (Princeton NJ: PUP)

[12] Einstein A and Rosen N 1937 On gravitational waves J. Franklin Inst. 22343

[13] Erdélyi A, Magnus W, Oberhettinger F and Tricomi F G 1954 Tables of integral transforms Vol. 2 Bateman Manuscript Project (New York: Mac Graw Hill)

[14] Gleiser J R, Nicasio C O, Price R H and Pullin J 2000 Gravitational radiation from Schwarzschild black holes: the second order perturbation formalism Phys. Reports $\mathbf{3 2 5}$ 41

[15] Gowdy R H 1971 Gravitational waves in closed universes Phys. Rev. Letters 27826

[16] Lindblom L and Brill D R 1974 Inertial effects in the gravitational collapse of a rotating shell Phys. Rev. D 103151

[17] Lynden-Bell D and Kalnajs A J 1972 On the generating mechanism of spiral structure Mon. Not. Roy. Ast. Soc. 1571

[18] Lynden-Bell D, Katz J and Bičák J 1995 Mach's principle from the relativistic constraint equations Mon. Not. Roy. Ast. Soc. 272 150, Erratum MNRAS 2771600

[19] Landau L D and Lifshitz E M 1959 The classical theory of fields (London: Pergamon) 
[20] Lyne A G 2006 The double-pulsar binary J0737-3039 Advances in Space Research 3812

[21] Mach E 1883 The science of mechanics (London: Open Court Pub. Co.) 1942 edition

[22] Manchester R N, Taylor J H and Huguenin G R 1975 Observations of pulsar radio emission. II - Polarization of individual pulses Astroph. J. 19683

[23] Mashhoon B, McClune J C and Quevedo H 2000 On a class of rotating gravitational waves Class. Quantum Grav. 17533

[24] McLaughlin M A, Lyne A G and 11 coauthors 2004 The Double Pulsar System J07373039: Modulation of A by B at Eclipse Astroph. J. 616 L131 (Reprint arXiv:astro$\mathrm{ph} / 0408297)$

[25] Morgan T and Bondi H 1970 Transfer of energy in General Relativity Proc. R. Soc. Lond. A 320277

[26] Newton I 1687 Philosophi Naturalis Principia Mathematica Translated by Andrew Motte 1729 (London: Roy.Soc.)

[27] Taylor J H, Fowler L A and McCulloch P M 1979 Measurements of general relativistic effects in the binary pulsar PSR 1913+16 Nature 277437

[28] Taylor J H and Weisberg J M 1982 A new test of general relativity - Gravitational radiation and the binary pulsar PSR 1913+16 Astroph. J. 253908

[29] Weber J General Relativity and gravitational waves 1961 (New York: Interscience)

[30] Weber J and Wheeler J A 1957 Reality of the cylindrical waves of Einstein-Rosen Rev. Mod. Phys. 29509

\section{A Appendix: Evaluation of the Integrals $I_{m}$ and $H_{m}$}

$$
I_{m}=\int_{0}^{u} u^{m} Q^{-m-3 / 2} d u \quad ; \quad H_{m}=\int_{u}^{\infty} u^{m-1} Q^{-m-3 / 2} d u .
$$

We write

$$
Q=u^{2}+2 b u+c^{2} \quad, \text { where } \quad b=1-\widetilde{t}^{2} \quad \text { and } \quad c=1+\widetilde{t}^{2},
$$


so $c \geq b$. Then

$$
\int_{0}^{u} Q^{-1 / 2} d u=\operatorname{arcsinh}\left(\frac{u+b}{\sqrt{c^{2}-b^{2}}}\right)-\operatorname{arcsinh}\left(\frac{b}{\sqrt{c^{2}-b^{2}}}\right)
$$

and

$$
\int_{0}^{u} Q^{-3 / 2} d u=-2 \frac{\partial}{\partial c^{2}}\left(\int_{0}^{u} Q^{-1 / 2} d u\right)=\frac{u+b}{c^{2}-b^{2}} Q^{-1 / 2}-\frac{b / c}{c^{2}-b^{2}} \equiv X(b, u) .
$$

Now differentiate $m$ times with respect to $b$ :

$$
(-1)^{m}(2 m+1) ! ! \int_{0}^{u} u^{m} Q^{-m-3 / 2} d u=\frac{\partial^{m} X(b, u)}{\partial b^{m}} .
$$

So

$$
I_{m}(u)=\frac{(-1)^{m}}{(2 m+1) ! !} \frac{\partial^{m} X(b, u)}{\partial b^{m}},
$$

where in this expression $c=1+\tilde{t}^{2}$ and, after differentiation $b=1-\tilde{t}^{2}$. Now from A.4

$$
X(b, \infty)=\frac{1-b / c}{c^{2}-b^{2}}=\frac{1}{c(c+b)}
$$

so

$$
I_{m}(\infty)=\frac{m !}{(2 m+1) ! !} \cdot \frac{1}{c(c+b)^{m+1}}
$$

For general $b$ and $u$

$$
\int_{u}^{\infty} Q^{-3 / 2} d u=\frac{1}{c^{2}-b^{2}}\left[1-(u+b) Q^{-1 / 2}\right]
$$

Differentiating with respect to $c^{2}$ and multiplying by $-2 / 3$ :

$$
\int_{u}^{\infty} Q^{-5 / 2} d u=\frac{1}{3\left(c^{2}-b^{2}\right)^{2}}\left[2-\frac{(u+b)\left(2 Q+c^{2}-b^{2}\right)}{Q^{3 / 2}}\right] \equiv Y(b, u) .
$$

Differentiating $m-1$ times with respect to $b$ we find

$$
(-1)^{m-1} \frac{1}{3}(2 m+1) ! ! \int_{u}^{\infty} u^{m-1} Q^{-m-3 / 2} d u=\frac{\partial^{m-1} Y(b, u)}{\partial b^{m-1}} .
$$

Hence,

$$
H_{m}=\frac{3(-1)^{m-1}}{(2 m+1) ! !} \frac{\partial^{m-1} Y(b, u)}{\partial b^{m-1}},
$$

again $b$ must be set equal to $1-\tilde{t}^{2}$ after the differentiation. From A.10 we see that

$$
Y(b, 0)=\frac{1}{3 c^{2}}\left[\frac{1}{c(c+b)}+\frac{1}{(c+b)^{2}}\right] .
$$

So

$$
(-1)^{m-1} \frac{\partial^{m-1} Y(b, 0)}{\partial b^{m-1}}=\frac{1}{3 c^{2}}\left[\frac{(m-1) !}{c(c+b)^{m}}+\frac{m !}{(c+b)^{m+1}}\right]
$$


and

$$
H_{m}(0)=\frac{(m-1) !}{(2 m+1) ! !} \cdot \frac{b+(m+1) c}{c^{3}(c+b)^{m+1}} .
$$

Whereas A.6 and A.12 give $I_{m}$ and $H_{m}$ for all values of $u$, A.8 and A.15 give their values at 0 and $\infty$, nevertheless it is useful to view their behaviours at small and at large $u$ explicitly. In particular for $\tilde{\rho}^{2} \ll \frac{1}{2}\left(1+\tilde{t}^{2}\right)$, the region encompassed by the wave but not 'feeling' it, we may expand $Q^{-m-3 / 2}$ in the form

$$
Q^{-m-3 / 2} \simeq \frac{1}{c^{2 m+3}}\left[1-(2 m-3) \cdot \frac{b u}{c^{2}}\right] \quad, \quad u \ll c .
$$

Then

$$
I_{m}(u) \simeq \frac{u^{m+1}}{c^{2 m+3}}\left[\frac{1}{m+1}-\frac{2 m-3}{m+2} \cdot \frac{b u}{c^{2}}\right] \quad, \quad u \ll c,
$$

and likewise

$$
H_{m}(u) \simeq H_{m}(0)-\frac{u^{m}}{c^{2 m+3}}\left[\frac{1}{m}-\frac{2 m-3}{m+1} \cdot \frac{b u}{c^{2}}\right] \quad, \quad u \ll c .
$$

Likewise for $\tilde{\rho}^{2} \gg \frac{1}{2}\left(1+\tilde{t}^{2}\right)$ which is $u \gg c$

$$
Q^{-m-3 / 2} \simeq \frac{1}{u^{2 m+3}}\left[1-(2 m-3) \frac{b}{u}\right] \quad, \quad u \gg c,
$$

so

$$
I_{m}(u) \simeq I_{m}(\infty)-\frac{1}{u^{m+2}}\left[\frac{1}{m+2}-\frac{2 m-3}{m+3} \cdot \frac{b}{u}\right] \quad, \quad u \gg c
$$

and

$$
H_{m}(u) \simeq \frac{1}{u^{m+3}}\left[\frac{1}{m+3}-\frac{2 m-3}{m+4} \cdot \frac{b}{u}\right] \quad, \quad u \gg c .
$$

Finally when $b=c, Q$ becomes a perfect square so the integrals are easier; since both $b$ and $c$ are then 1 we express the answer that way:

$$
I_{m}=\sum_{s=0}^{m}(-1)^{m-s}\left(\begin{array}{c}
m \\
s
\end{array}\right) \frac{(1+u)^{s-2 m-2}-1}{(s-2 m-2)} \quad, \quad H_{m}=\sum_{s=0}^{m-1}(-1)^{m-s}\left(\begin{array}{c}
m \\
s
\end{array}\right) \frac{(1+u)^{s-2 m-2}}{(s-2 m-2)} .
$$


fig. $5 a: \frac{\psi}{B}$ at $\tilde{t}=-2$

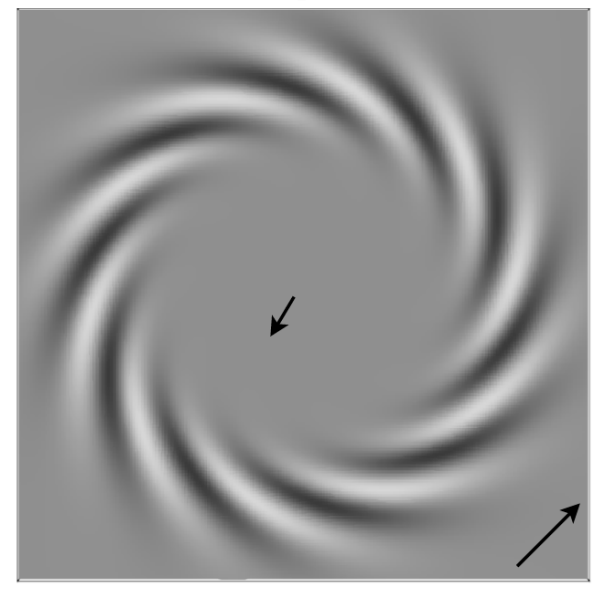

fig. $5 c: \frac{\psi}{B}$ at $\tilde{t}=0$

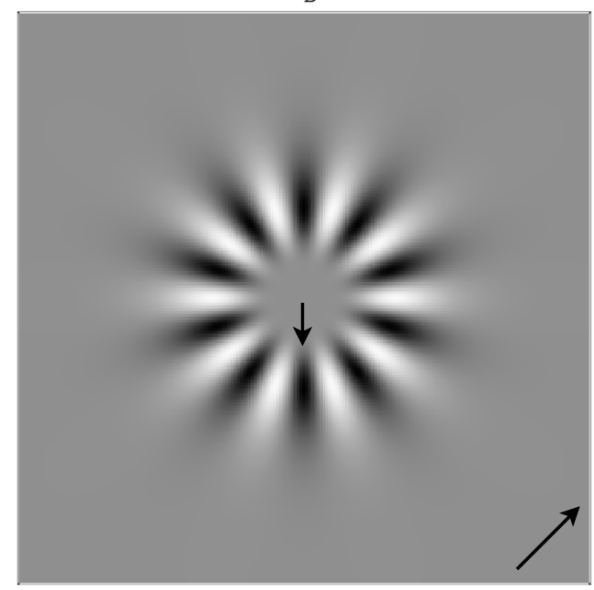

fig. $5 e: \frac{\psi}{B}$ at $\tilde{\mathrm{t}}=2$

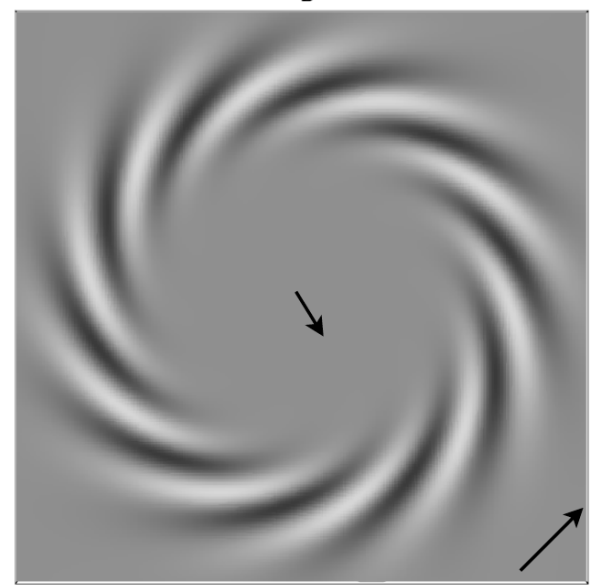

fig. $5 b: \frac{\psi}{B}$ at $\tilde{t}=-1$

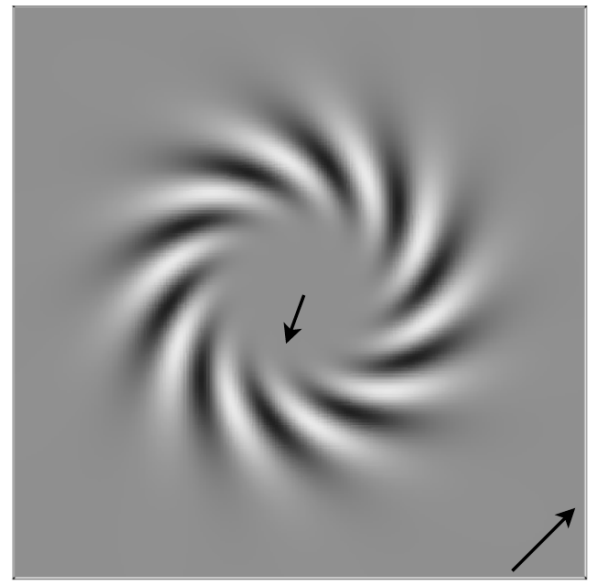

fig. $5 \mathrm{~d}: \frac{\psi}{B}$ at $\tilde{t}=1$

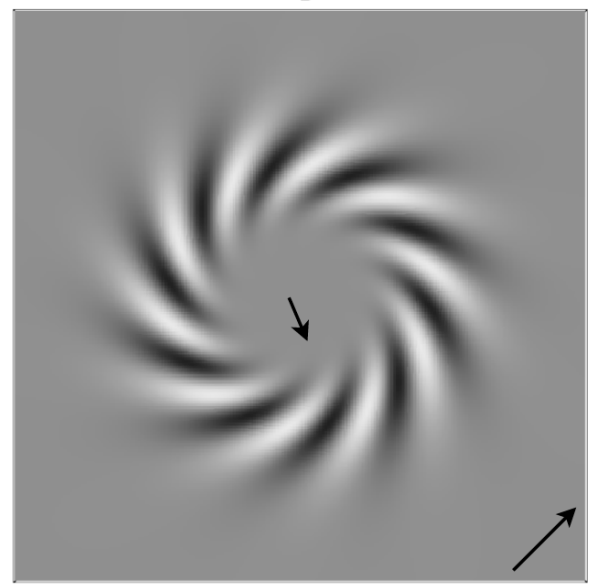

fig. $5 f: \frac{\psi}{B}$ at $\tilde{t}=10$

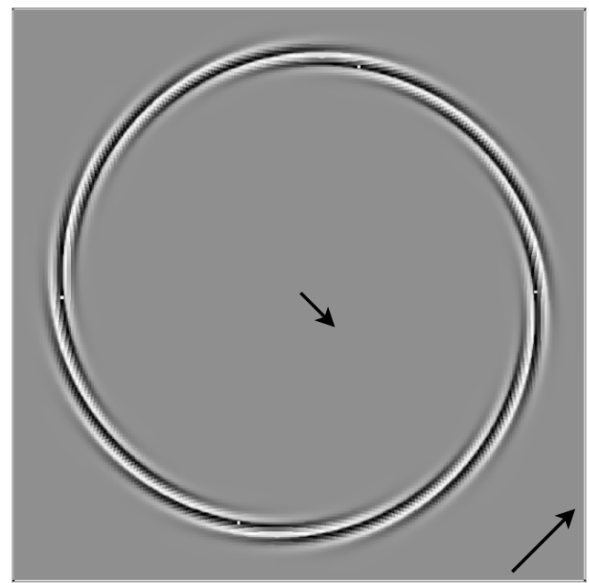

Figure 5: This shows the $m=10$ wave which always rotates anticlockwise. As it comes inwards (Figure 5a) at $\tilde{t}=-2$ it is in the form of a leading spiral with the outer parts of the arms ahead of the central parts. By $\tilde{t}=-1$ (Figure 5b) the spiral has started to open. By $\tilde{t}=0$ (fig.5c) the central parts have caught up and the spiral has changed to a cartwheel structure but rotation keeps it beyond $\tilde{\rho} \simeq 0.4$. By $\tilde{t}=1$ (Figure $5 \mathrm{~d}$ ) the spiral has become trailing as befits a wave that now feeds angular momentum outwards. By $\tilde{t}=2$ (Figure 5e) the spiral becomes tighter and the flat central cylinder becomes larger. We show $\tilde{t}=10$ (Figure 5f) at a small scale but notice the beautiful tight wrapping of the narrow arms. Also notice the opposite spirality of the conjugate pairs $\tilde{t}= \pm 2$ and $\tilde{t}= \pm 1$. The figures encompass a radius $\tilde{\rho} \simeq 7(\tilde{\rho} \simeq 17$ for $\tilde{t}=10)$. The height of $\psi / B$ reduced by a factor of $10^{-4}$ 